\title{
A Brainwave Signal Measurement and Data Processing Technique for Daily Life Applications
}

\author{
Yoshitsugu Yasui \\ Advanced Technology Research, NTT DoCoMo
}

\begin{abstract}
A technique for measuring the psychophysiological status of the human and associated applications based on normal brain signals are examined and evaluated. A small single-point dry electrode developed for mobile use can capture brainwave activity from among dense external and internal electrical noise, and subsequently extract targeted frequency components. Continuous measurements during day and night provide a brainwave profile including wake and sleep states that can consistently explain states of human awareness. Statistical evaluation provides psychophysiological state change patterns which can be used to distinguish levels of alertness so as to prevent or avoid hazardous situations. We have taken a typical daily activity, namely, driving a car, to examine the applicability of our proposed method. Test results in terms of brain wave state show that the pattern while driving is changed by specific activity such as when talking on a mobile phone. J Physiol Anthropol 28(3): 145-150, 2009 http://www.jstage.jst.go.jp/browse/jpa2
\end{abstract}

[DOI: $10.2114 /$ jpa2.28.145]

Keywords: EEG, brainwave, mobile instrument, life monitoring, car driving

\section{Introduction}

Brain activity is an essential key to understanding the psychophysiological states of humans. Using non-invasive electroencephalograms (EEG) with a dry electrode at the forehead, brain states can be measured and analyzed without complex medical procedures. Although the brain is the most complex organ, advancements in brain science have produced theories on the relationships between brainwave characteristics and psychophysiological states. The challenge is to consolidate accurate measurements to capture subtle signals using simple instrumentation, then interpret the data into meaningful signatures. Presuming such applications are used during everyday activities, a small and lightweight sensor system must be developed and applied for testing.

With the advancements of brain science, fMRI (Functional
Magnetic Resonance Imaging) or infrared spectroscopes provide accurate interpretation of brain activity on brain nerve location (Haruno et al., 2004). This requires massive instrumentation and places several constraints on the subject. The conventional multi-point EEG requires a special headset and electrolyte gel that is unpleasant, which may lead to discomfort and result in the collection of erroneous data if applied during normal daily activity. Therefore, a lightweight, dry single-point EEG contact needs to be developed to solve this problem.

An EEG is easily affected by electrical potentials generated by muscle contractions (Quiroga, 2006). Using a single-point EEG sensor to observe electrical potentials, it is difficult to completely segregate brainwaves from these electrical signals. However, from a physiological viewpoint, an irritation or nervous condition can be detected by observing rapid muscle movement of the eyes or facial tissues. Therefore, if it is possible to establish a rational interpretation for an input that includes a mix of brainwave and signals created by muscle movement for recognizing a certain physiological state, the EEG measurement will become more common in normal daily life.

The first attempt to understand the capability of the method is to determine sensor output during wake and sleep states, two different psychophysiological states of the brain.

The proposed measurement system and statistical approach are examined to differentiate sleep/awake states. Rapid Eye Movement (REM)-nonREM sleep cycles (Aserinsky and Kleitman 1953) are also discussed in terms of detection capability by this approach.

This exploratory exercise is to extract a signature that can be used as a precursor for hazard detection and prevention, as demanded by activities, such as driving a car, that require several concurrent brain states, including concentration, relaxation, focus, and alertness (Harada et al., 2007).

A small wearable single-point EEG probe, along with a mobile device are ideal for conducting such measurements while driving or performing in other real life situations. The absolute magnitude of brainwaves measured by an EEG sensor varies with time, sensor location, and skin condition. The 
consideration is therefore to analyze a portion of each frequency component during each time period, with the assumption being that dominant frequency components can represent certain brain states. If frequency characteristics based on that assumption can match physiological state recognition, a single-point mobile EEG sensor becomes an effective monitoring probe for real-life application. The capability of this method is examined by monitoring subjects throughout the course of a day and extracting crucial information towards realizing the stated objective.

\section{Method}

The EEG sensor (ThinkGear, NeuroSky, CA, USA) used for this test consisted of a pea-sized $(10 \mathrm{~mm}$ diameter) active electrode and reference electrode to measure the electrical potential of the two points. The electrical potential is supplied directly to the embedded chipset for analog filtering with band pass and notch filters and $128 \mathrm{KHz}$ digital sampling at $\mathrm{A} / \mathrm{D}$ converter. The digitized data are analyzed by FFT in the headset circuit board to produce the power value of each frequency component and the results are transmitted to the cell phone. Using the on-board computation and signal process circuitry, the unit can acquire highly sensitive brainwave data without being contaminated by noise. The sensor is attached to the head of the subject with a common headband apparatus, as shown in Fig. 1. A photograph of the headset is presented in Fig. 2. The electrode and electronics for data processing have a barely noticeable weight in addition to the fabric headband. Much of the weight is dominated by the Bluetooth unit and its battery. The reference is taken at the lobe and the circuitry is also loaded on the headband. Data are captured and presented in each frequency band: Delta $(0-3 \mathrm{~Hz})$, Theta $(4-7 \mathrm{~Hz})$, Alpha $(8-12 \mathrm{~Hz})$, Low Beta $(12-15 \mathrm{~Hz})$, Mid Beta $(16-20 \mathrm{~Hz})$ and High Beta $(21-30 \mathrm{~Hz})$ every two seconds.

The system monitors electrical potential between the active and reference electrodes which are attached on the forehead and the earlobe, respectively. To minimize discomfort and interference with natural movement and behavior, data were transmitted via wireless connection by Bluetooth and stored in the memory of a cell phone located beside the subject anywhere Bluetooth can be connected wirelessly to the headset unit. Due to limited battery life, measurements were temporarily halted within 8 hours to allow battery replacement or recharging. Alternative systems requiring less power can also be substituted for Bluetooth.

The output from the EEG sensor is subjected to frequency component analysis. Although the extracted data represent the electric potential difference between active and reference electrodes, its amplitude does not have a special meaning. However, analysis to examine the power ratio of the frequency component to total power can show which frequency range is dominant at the time the data are taken.

Since frequency component analysis often suffers from high electrical activity including ocular artifacts (OA), especially

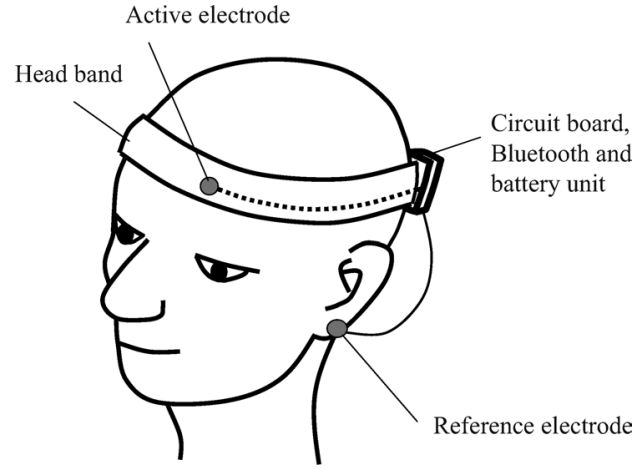

Fig. 1 Measurement instrumentation illustration showing the headband implementation of sensor nodes, electric circuitry, and wireless transmitter.

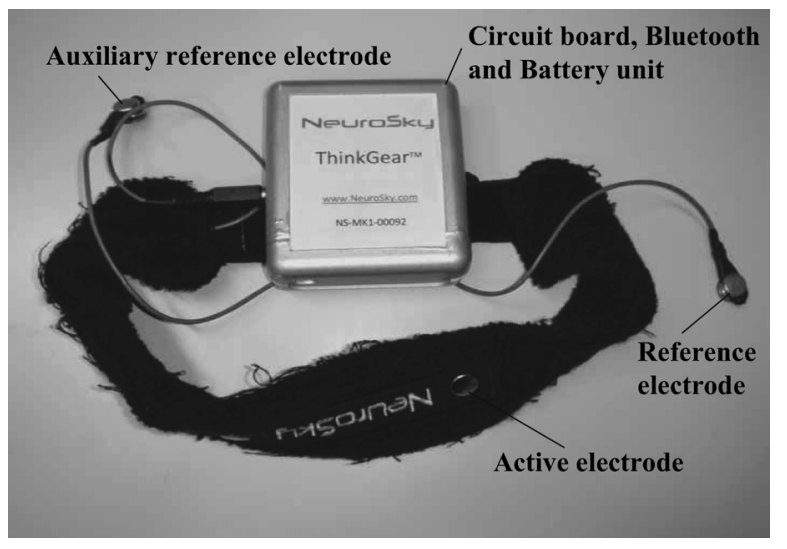

Fig. 2 Picture of the headset instrumentation.

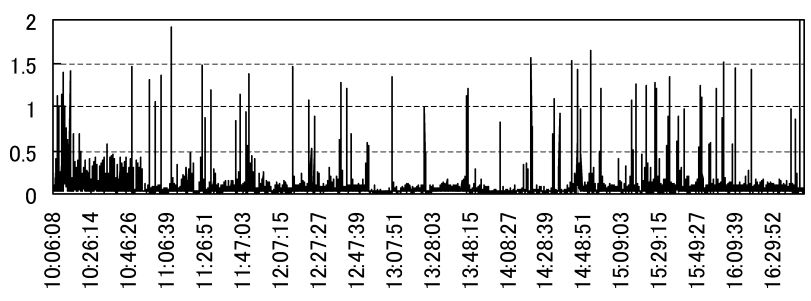

Fig. 3 Absolute magnitude of the signals during long-term measurement.

higher frequency components have less amplitude and hide in statistics, we eliminated excessive large responses of more than 2 sigma variation of the amplitude as artifacts in these tests. The amplitude distribution is presented in Fig. 3.

Although several methods are proposed (Hass et al., 2003; Krishnaveni et al., 2006), it is difficult to separate artifacts such as OA from EEGs completely. In a series of measurements, even ignoring a small number of data which exhibit excessive responses supposedly by the artifact contaminated, since the measurement is focusing on the frequency components continuously being produced in the psychophysiological state, the statistical representation by the rest of the data can exhibit the gross characteristics of the state. The comparison based on the frequency component 
ratio is also less vulnerable than absolute values, which are contaminated by ambient and body-induced noise.

Attempting to adopt a statistical approach with the data in a certain time period is to understand the physiological status signature, which, in general changes slowly (Hastings, 1998; Louise et al., 2007), rather than to find mind orientations by collecting specific brain waves. The evidence sought here will be a series of brain activities which differentiate certain physiological states in the exposure of a specific situation or when engaging in an activity such as driving a car.

\section{Results}

Two types of tests are chosen to examine the applicability to real life measurement of brain waves. The first attempt is a continuous measurement during normal life including nighttime sleep. The other is a specific application for car driving. For the continuous measurement, the subject is a male student aged 22. The measurements were done on a normal college day and that night. For car driving, the subject is a male aged 30 , who has 10 years' driving experience. Since the purpose of this paper is to show the measurement methodology and feasibility, the typical test results are presented.

\section{Continuous measurement in a student's normal life}

The result of daytime measurements is graphically shown in Fig. 4. This exhibits a slow cycle that transitions from higher frequency dominance; around 10:30 to 12:00, to lower frequency dominance; around 13:00 to 15:00, and again back to higher frequency; around 15:00 to 17:00. Observing the subject's daily activity, it represents an active morning, followed by a lunch break, return to afternoon work, bouts of drowsiness, and a second wind in the late afternoon. The subject reported his awareness during the test at the post-test interview. That indicated concentration in the morning class, relaxation during lunch, being somewhat drowsy at the beginning of the afternoon class, then concentration in the later class, and relaxation again after school enjoying conversation with friends to the end of the measurement period.

The results of night-time measurements while the subject slept are shown in Fig. 5. Lower frequency components occupy major portions of the sleep period. Also, the active highfrequency components were observed every two to three hours in Fig. 5. The research of sleep state including REM/nonREM is one of the potential applications.

Data vulnerability against noise and artifacts is one issue that requires addressing. Noise characteristics can be defined by ambient measurements without mounting the sensor to the forehead. The measurement output is continuously monitored, including the sensor-dismounting period. A none-specific frequency dominant random distribution characteristic is shown after 13:00 in Fig. 6, where the sensor was detached from the head. The frequency distribution is apparently different when compared with Fig. 4, which doesn't have a sensor-detached period. At 15:30 the sensor was placed back

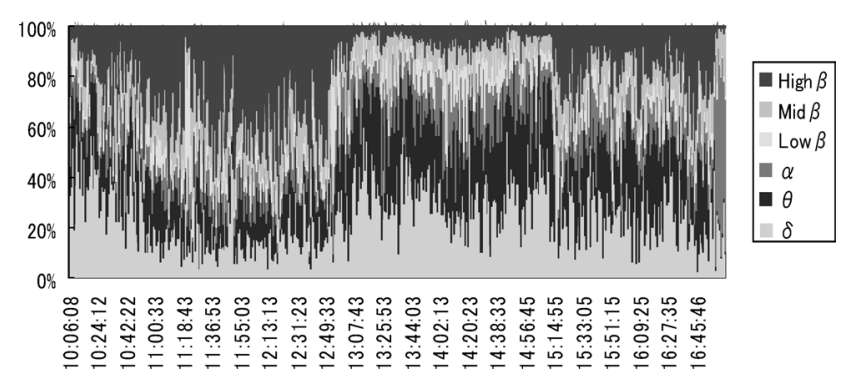

Fig. 4 Brain wave frequency component distribution of the daytime measurement, showing the dominance relative to the total power.

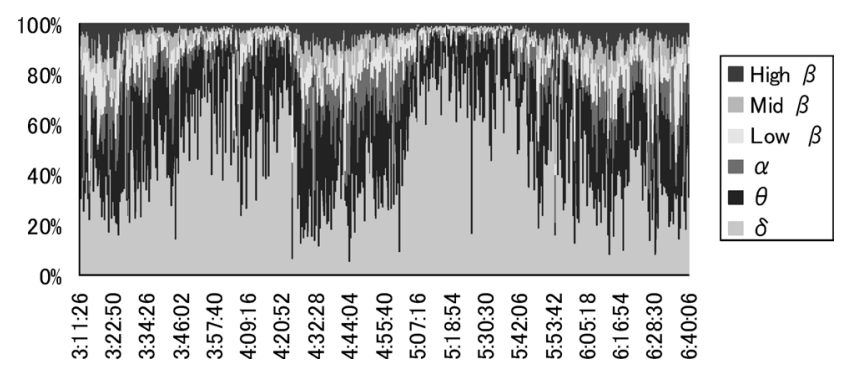

Fig. 5 Brain wave frequency component distribution of the measurement during night time including sleep, showing the dominance relative to the total power.

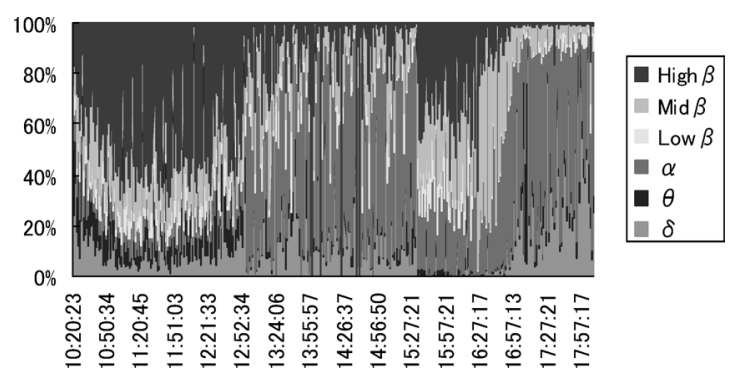

Fig. 6 Brain wave frequency component relative distribution of the measurement with detached sensor during 13:00-15:30, showing the dominance relative to the total power.

on the forehead, and the signal shows a similar frequency component distribution pattern to that observed before the detachment.

From collecting data in the continuous measurements, it can be assumed that the frequency component distribution pattern of the EEG measurement can reveal obvious differences during the daily activities in a relative fashion, although brain wave signals are weak, masked by noises or muscle movement, and show variations depending on sensor locations and surrounding conditions.

\section{Car driving}

The activity for driving a car can elicit various brain responses, such as nervousness, concentration, relaxation, focus, and sometimes drowsiness. Especially if we can extract an unusual condition and create a specific frequency-dominant component, it can be useful for early notification of a specific 
condition and/or for hazard prevention.

To check how the brain wave sensor signal is exhibited during normal driving measurements are first taken for several hours of driving. It may be difficult to correctly extract a certain difference during the driving period since several types of stimuli may induce a variety of signals reflecting concentration, relaxation, tension, or a combination of them. Therefore, frequency component distribution during the entire driving period is observed and then compared with a typical driving occasion. We selected a phone conversation as a typical event that would interrupt the concentration level of the driver (Parkes et al., 2007; Redelmeier and Tibshirani, 1997). The driver was monitored to determine whether the phone call changed the brain wave patterns. It may be possible by monitoring driving performance in the interruption, but this introduces a number of variables, such as driver's skill, driving scenario, and difficulties, that complicate the evaluation process and results. This lightweight wearable dry EEG sensor and monitoring system can minimize the driver's level of discomfort, which can cause an additional stimulus to the brain activity and deteriorate the data.

To ensure that the test scenario represents normal driving conditions, reference data were collected on public roads. For safety reasons, the driver talked with a hands-free device. Although excluding handheld talking for this test, which is a matter of public concern, certain accident research (McEvoy et al., 2005) has indicated no difference between handheld and hands-free devices. Data for 20 minutes were sampled for the interval to ensure the statistical significance of the data. To clarify the brain state change, the consecutive data of pre- and post-intervals of the phone conversation were measured.

The Alpha frequency components and Beta frequency component ratio were used to examine the mental concentration level change. In this calculation, three Beta components were combined into a $12-30 \mathrm{~Hz}$ single component in equally balance with Alpha to avoid a numerical calculation truncation error.

The data in Table 1 indicate that cellular phone conversations during driving produce a different signature for the Alpha frequency component and Beta frequency component ratio. Two tests show the same trend. The Student t-tests show statistically significant differences between paired test data as shown in Table 2. The difference of degrees of freedom is due to the extreme data elimination mentioned above and the contingency such as discontinuation as the result of a traffic jam. The $p$-value between the pre- and post-data of the second test shows $p>0.5$. This implies that there is no significant difference in driving condition between the pre- and post-data in terms of brain activity. Even though the first test shows some difference between the pre- and post-data supposedly due to the different number of measurement points, the $p$-value paired with pre- and post-data shows a much greater similarity than when paired with the phone conversation data, which is the same as the second test.

Driving while talking on the phone seems to suppress the
Table 1 Comparisons of Alpha/Beta power ratio between normal driving and driving with cell-phone talking from 20 minutes' consecutive measurement

\begin{tabular}{cccc}
\hline Tested conditions & Samples & Mean & S.D. \\
\hline $1-1$ & 608 & 0.7252 & 0.6836 \\
$1-2$ & 605 & 1.2935 & 0.9618 \\
$1-3$ & 258 & 0.8990 & 0.6873 \\
$2-1$ & 636 & 0.5706 & 0.4873 \\
$2-2$ & 621 & 0.7343 & 0.6095 \\
$2-3$ & 610 & 0.5864 & 0.5858 \\
\hline
\end{tabular}

1-1: Pre 20 minutes driving, First test.

1-2: 20 minutes driving with phone call, First test.

1-3: Post 20 minutes driving, First test.

2-1: Pre 20 minutes driving, Second test.

2-2: 20 minutes driving with phone call, Second test.

2-3: Post 20 minutes driving, Second test.

S.D.: Standard Deviation.

Table 2 Results of the significance of differences by Student t-test between with/without cell-phone talking; t-value is conditioned by unequal samples and unequal variance

\begin{tabular}{lrrc}
\hline Tested conditions & t-value & d.f. & $p$-value \\
\hline$(1-1)$ vs (1-2) & 11.90 & 1089 & $p<0.000000$ \\
$(1-2)$ vs (1-3) & 6.81 & 667 & $P<0.000000$ \\
$(1-1)$ vs (1-3) & 3.41 & 482 & $p=0.000704$ \\
$(2-1)$ vs (2-2) & 5.30 & 1195 & $p<0.000000$ \\
$(2-2)$ vs (2-3) & 4.30 & 1228 & $p=0.000018$ \\
$(2-1)$ vs (2-3) & 0.51 & 1195 & $p=0.610146$ \\
\hline
\end{tabular}

d.f.: degrees of freedom.

output of Alpha frequency components versus Beta frequency that is different from normal driving, in which several situations create a variety of frequency components. It can be conceived that the actual content of the conversation may also cause different brain frequency patterns. Although these issues are not addressed in this paper, we suppose the 20 minutes is long enough to express a variety of content and makes the conversation an average and standard phone conversation.

\section{Discussion}

Although the system is exposed to white noise acting equally over the entire frequency range, as observed in Fig. 4 and Fig. 6 when the sensor is detached from the head, the method based on the relative dominance of each component can cancel this effect out.

Muscle contractions may affect specific frequencies in brainwaves. However, if registered as an enduring constant cyclical motion, it may also be dealt with as a physiological signature, since it is produced by an evoked event. For instance, assuming that REM sleep exhibits high frequency components created by brainwaves and OA, the result of the frequency power density ratio analysis shows specific pattern changes, which can be used for sleep state analysis including 
REM and nonREM sleep cycles. The small instrumentation and method developed here enable natural sleep monitoring without any inconvenience. More instrumentation and analysis may be necessary to examine the detailed diagnostic of sleep conditions, but for sleep pattern recognition this provides a monitoring capability.

Not only pertaining to the sleep state, the day-long monitoring shows a certain profile exhibiting physiological brain state changes in normal life. The result shows that the proposed measurement technique obviously exhibits frequency component dominance change. During a student's regular school day, higher frequencies dominated in the morning class, more low frequencies were shown after lunch, higher frequencies came back in the afternoon class and higher frequencies recede when school is dismissed.

Rather than capturing instantaneous brainwave signals and diagnosing them, we chose to monitor over a certain time period the distribution and dominance of the frequency components to find a statistical signature from a shift in frequency characteristics. In that sense, the goal of this measurement is to recognize brain-induced signals which can be evaluated to differentiate psychophysiological states when examining the level of mental fitness for each human activity.

For car driving, what can be observed is that the driver exhibits and repeats a different signature when using a cell phone during driving, creating higher frequency components data, the frequency of which are related to awareness and alertness in brainwaves. It is expected that these results support the long-held belief that using a phone while driving is a potential hazard.

Further measurements and comprehensive analysis are necessary to consolidate the overall applicability of this method towards multiple events and subjects. However, our results indicate that the subject's brain activity is overwhelmed by using the telephone while driving. The quantification of driving danger and the associated brain state is beyond the scope of this paper and may require a more direct evaluation. It is noted, however, that this evaluation does not delve into a person's ambiguous feelings or performance level, but addresses only the notion that brain signals may indicate essential signatures that govern human activity.

The test instruments and methodology implemented here can be extended to several applications to further understand human interaction while driving. For example, it can be used for automotive or transportation facility design to circumvent stressful situations. Since the system only requires a small sensor placed on the forehead and a wearable circuit that transmits data wirelessly, it can easily be applied to driving tests without causing any discomfort or physical constraints that may deteriorate the results.

In conclusion, the noninvasive noise-controlled singlechannel dry sensor was able to extract brainwave signal components at various frequency bands. The data become more expressive after applying statistical processes than just by focusing on instantaneous responses. The mobility of the system achieved effortless measurements during normal daily life without constraints, extending measurement possibilities and directly bridging biological signals and normal human behavior. Applied specifically to driving a vehicle, the data showed an obvious pattern change when a mobile phone was introduced, even though the measurements are limited. We expect this neuroscientific approach will assume a useful role in assessing normal daily human activities, especially for matters of public concern including the risk associated with driving while talking on a phone. The tiny, wearable brainwave sensor and its system will provide further information through real-time life measurements and related applications.

Acknowledgements The author thanks Dr. KooHyoung Lee, NeuroSky Inc., and Mr. Kevin Williams for reading the manuscript, and Mr. Tansheng $\mathrm{Li}$ and Prof. Noriyoshi Yamauchi, Waseda University for helping with the tests.

\section{References}

Aserinsky E, Kleitman N (1953) Regularly occurring periods of eye motility, and concomitant phenomena, during sleep. Science 118: 273-274

Cui R, Li B, Suemaru K, Araki H (2007) Differential effects of psychological and physical stress on the sleep pattern in rats. Acta Med Okayama 61: 319-327

Harada H, Nishihara H, Morizumi K, Ota H, Hatakeyama E (2007) A comparison of cerebral activity in the prefrontal region between young adults and the elderly while driving. Physiol Anthoropol 26: 409-414

Haruno M, Kuroda T, Doya K, Toyama K, Kimura M, Samejima K, Imamizu H, Kawato M (2004) A neural correlate of reinforcement-based behavioral learning in caudate nucleus: An fMRI study of a stochastic decision task. Neurosc 24: 1660-1665

Hass SH, Frei MG, Osorio I, Pasik-Duncan B, Radel J (2003) EEG ocular artifact removal through ARMAX model system identification using extended least squares. Communications in Information and Systems 3: 19-44

Hastings MH (1998) The Brain, Circadian Rhythms, and Clock Genes. BMJ 317: 19-26

Insurance Institute for Highway Safety (2005) The 1st Evidence of Effects of Cell Phone Use on Injury Crashes: Crash risk is four times higher when driver is using a handheld cell phone, News release note of Insurance Institute for Highway Safety. http://www.iihs.org/

Krishnaveni V, Jayaraman S, Aravind S, Hariharasudhan V, Ramadoss K (2006) Automatic identification and removal of ocular artifacts from EEG using wavelet transform. Measurement Sci Rev 6-2: 45-57

Louise V, Fairclough SH (2007) Establishing the Psychophysiological Variables that can Identify and Predict Operator Subjective State, Human Factors and Ergonomics Society Annual Meting Proceedings. Aerospace Systems 5: 90-94 
McEvoy SP, Stevenson MR, McCartt AT, Woodward M, Haworth C, Palamara P, Cercarelli R (2005) Role of mobile phones in motor vehicle crashes resulting in hospital attendance: a case-crossover study. Bri Med J (BMJ online) http://www.bmj.com

Parkes AM, Luke T, Burns PC, Lansdown T (2007) Conversations in cars: the relative hazards of mobile phones. Transportation Laboratory report TRL664, UK

Quiroga RQ (2006) Evoked Potentials, Encyclopedia of Medical Devices and Instrumentation. 2nd ed., John Wiley and Sons, Inc., 233-246

Redelmeier DA, Tibshirani RJ (1997) Association between cellular-telephone calls and motor vehicle collision. The New Eng J Med 336: 453-458

Yasui Y, Tian Q, Yamauchi N (2008) A data process and wavelet analysis method used for monitoring daily physiological attributes, The Proc IEEE Engineering in Med Biol Conference, Vancouver, 1447-1450

Webster G (2006) Encyclopedia of Medical devices and Instrumentation. 2nd ed., John Wiley \& Sons, Inc., 233-246

Received: November 14, 2008

Accepted: February 25, 2009

Correspondence to: Yoshitsugu Yasui, Advanced Technology Research, NTT DoCoMo, 2-11-1 Nagatacho, Chiyoda, Tokyo 100-6150, Japan

Phone: +81-03-5156-1745

Fax: +81-03-5156-0232

e-mail: yasuiy@nttdocomo.co.jp 